\title{
Two Aspects of Cardiac Alternans - Difference and Correlation between Them
}

\author{
Wei Wang ${ }^{1}$, Dominic G Whittaker ${ }^{1}$, Haibo $\mathrm{Ni}^{1}$, Kuanquan Wang ${ }^{2}$, Henggui Zhang ${ }^{1}$ \\ ${ }^{1}$ University of Manchester, Manchester, UK \\ ${ }^{2}$ Harbin Institute of Technology, Harbin, China
}

\begin{abstract}
Cardiac alternans are observed as action potential duration (APD) alternans and/or $\mathrm{Ca}^{2+}$ transient (CaT) alternans. This study aims to investigate the mechanism underlying the genesis of the two types of alternans, their difference and correlation. Two human ventricle models were used for generating cardiac alternans. APD restitution and CaT-SR content curves were constructed to investigate possible different mechanisms. Results showed that alternans produced by the ORd model sustained with significant CaT but no obvious APD alternans, whereas alternans generated by the TP model manifested as obvious APD but insignificant CaT alternans. CaT alternans in the ORd model was correlated with a much steeper CaT-SR content curve, while the APD alternans in the TP model was correlated with a steeper APD restitution curve. Furthermore, $I_{C a L}$ clamp simulations showed the magnitude of $I_{C a L}$ was crucial for generating the CaT alternans as a large $I_{C a L}$ could change its alternating status. Our results suggest that each of the APD or the CaT alternans has its own primary mechanism, and sustained CaT alternans is not necessarily related to APD alternans.
\end{abstract}

\section{Introduction}

Cardiac alternans, which mainly manifest as beat-tobeat oscillations in T-wave interval and the strength of cardiac muscle contraction on clinical diagnosis, is a well-known sign for predicting cardiac arrhythmia. At the cellular level, cardiac alternans is always observed in two aspects: one exhibited as action potential duration (APD) alternans and the other as intracellular $\mathrm{Ca}^{2+}$ transient (CaT) alternans. It is believed that APD and CaT alternans are closely linked, since they always occur at the same on-set time and have similar variation trends. The complicated feedback system between APD and CaT alternans makes it hard to determine which one is the primary mechanism of cardiac alternans, leaving it still a controversial problem.

Previously, cardiac alternans was believed to be attributable to the refractory properties of myocytes.
When myocytes are paced quickly, the increased pacing rate leads to a decreased diastolic interval (DI). As a result, the membrane currents, especially $\mathrm{I}_{\mathrm{Na}}$, will not have enough time to recover from their inactivated states, which leads to reduced current in the next AP, producing a smaller AP. This mechanism is typically manifested by a steep APD restitution curve [1], with a slope usually $>1$. However, recent studies have found APD alternans can also occur with a flat restitution curve, and others have even found CaT alternans occurring without significant APD alternans $[2,3]$. These studies support the $\left[\mathrm{Ca}^{2+}\right]_{\mathrm{i}} \rightarrow$ $\mathrm{V}_{\mathrm{m}}$ hypothesis, which postulates that instabilities in intracellular $\mathrm{Ca}^{2+}$ cycling processes are the primary mechanism of cardiac alternans while APD alternans is the secondary result.

This study aims to investigate the mechanism underlying the genesis of the two aspects of alternans, by examining their difference and correlation using two human ventricular cell models. Simulation results suggest that these two types of cardiac alternans may have their own primary mechanisms and CaT alternans plays a key role on sustained cardiac alternans.

\section{Methods}

In this study, two human ventricle models were implemented for generating cardiac alternans. One is the ten-Tusscher model [4] modified by Adeniran et al. [5] (the TP model), and the other is the O'Hara-Rudy dynamic model [6] (the ORd model). The two models have been widely used in cardiac simulations.

An S1-S2 protocol was used to elucidate the restitution properties of the two ventricle models. With $\mathrm{S} 1, \mathrm{BCL}=$ $1000 \mathrm{~ms}$ was used to stimulate the models until a steady state was reached. Then a second stimulus (S2) was given with a variable time of delay, producing variant DIs. This process was repeated for a range of DIs and the $\mathrm{APD}_{90}$ for the S2 stimulus was recorded to construct the APD restitution curve.

CaT-SR content curves were plotted to study the relationship between the SR content and CaT alternans. The simulations followed the experimental steps in [7]. The concentration of $\mathrm{Ca}^{2+}$ in SR content was firstly emptied, and then simulations ran at fast pacing rate 
$(\mathrm{BCL}=320 \mathrm{~ms}$ for the TP model and BCL $=250 \mathrm{~ms}$ for the ORd model) for 200s. The systolic intracellular and SR content concentration of $\mathrm{Ca}^{2+}$ were recorded for CaTSR content curve.

To study whether the amplitude or alternating $\mathrm{I}_{\mathrm{CaL}}$ current is the crucial determinant to generate CaT alternans, $\mathrm{I}_{\mathrm{CaL}}$ clamp protocols were implemented in the ORd model. In our previous simulation studies (not shown in this paper), we found that different stimulus strengths could influence the range of BCL that generates CaT alternans. That means that in the ORd model, the same BCL could either produce alternans or not dependent on different stimuli. In this study, BCL was set to be $250 \mathrm{~ms}$. In simulations, I $\mathrm{C}_{\mathrm{CaL}}$ current time traces were recorded during normal and alternans APs, denoted as $\mathrm{I}_{\mathrm{CaL} \_ \text {alter }}$ and $\mathrm{I}_{\mathrm{CaL} \_n o r m a l}$, with the amplitude of $2.5 \mu \mathrm{A} / \mu \mathrm{F}$ and $1.5 \mu \mathrm{A} / \mu \mathrm{F}$ respectively. Then the recorded $\mathrm{I}_{\mathrm{CaL}}$ was applied to clamp the $\mathrm{I}_{\mathrm{CaL}}$ current in the ORd model continuously to see if alternans could be generated or not. During the clamp time course, $\mathrm{I}_{\mathrm{CaL}}$ currents were kept identical beat-to-beat, therefore, there were no $\mathrm{I}_{\mathrm{CaL}}$ alternations during the clamp simulations.

\section{Results}

\subsection{Alternans generated by two human ventricle models}

By increasing the pacing rate, both the TP and ORd models began to generate beat-to-beat alternans in both APD and CaT amplitude at BCL $=350 \mathrm{~ms}$ and BCL $=$ 295ms, respectively. We recorded AP and CaT alternans at BCL $=320 \mathrm{~ms}$ for the TP model and BCL $=250 \mathrm{~ms}$ for the ORd model specifically for detailed analysis, since these two BCL ranges gave a relatively stable and
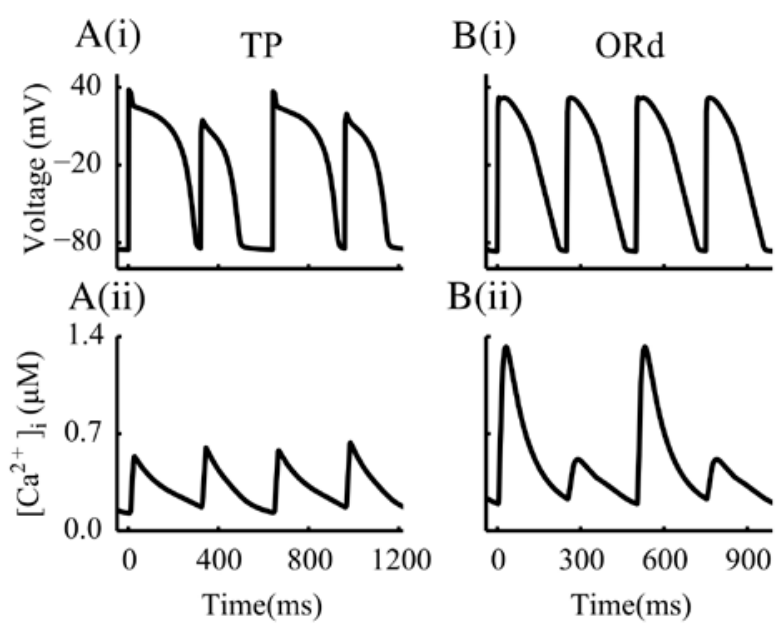

Figure 1. Simultaneously recorded APs (A(i)) and CaTs (A(ii)) at BCL $=320 \mathrm{~ms}$ for TP model; Simultaneously recorded $\mathrm{APs}(\mathrm{B}(\mathrm{i}))$ and $\mathrm{CaTs}(\mathrm{B}(\mathrm{ii}))$ at $\mathrm{BCL}=250 \mathrm{~ms}$ for ORd model.
Table 1. Different characteristics of cardiac alternans generated by the TP model and the ORd model.

\begin{tabular}{lll}
\hline Characters & TP & ORd \\
\hline$\Delta$ APD $(\mathrm{ms})$ & 123 & 12 \\
$\begin{array}{l}\Delta \text { Systolic }\left[\mathrm{Ca}^{2+}\right]_{\mathrm{i}}(\mu \mathrm{M}) \\
\begin{array}{l}\text { Sustained } \\
\text { Stable }\end{array}\end{array}$ & 0.06 & 0.82 \\
$\begin{array}{l}\text { Maximum slope of APD } \\
\text { restitution curve }\end{array}$ & No & Yes \\
$\begin{array}{l}\text { Maximum slope of CaT- } \\
\text { SR content curve }\end{array}$ & 1.82 & 0.35 \\
\hline
\end{tabular}

apparent cardiac alternans.

It was clear that cardiac alternans generated by the two models exhibited great differences (Figure 1). Compared with the ORd model, APD alternans produced by the TP model were much more significant. However, systolic CaT changed slightly in the TP model during the large AP alternations. Conversely, APD and CaT alternans generated by the ORd model exhibited little changes in AP morphologies and large variations in $\mathrm{CaT}$ amplitudes. In addition, alternans generated by the TP model only lasted for several beats, as the ratio of the large and small action potentials gradually decreased and eventually became zero. Unlike the TP model, the ORd model could keep alternating without ratio changes, which means this kind of alternans can sustain for a long time. Moreover, the two models started to generate alternans at different time points. The on-set time of alternans for the TP model was the beginning of fast pacing, whereas that for the ORd model was nearly 100s later. This is believed to be due to the level of $\mathrm{Ca}^{2+}$ concentration in SR content, which we will discuss in following sections.

Table 1 presents more detailed data of the alternans produced by the two models. It can be seen that cardiac alternans generated by the two models are completely different to each other. Thereby, we suggest that the two types of cardiac alternans have their own primary mechanisms and are correlated with each other through intracellular $\mathrm{Ca}^{2+}$ concentration. Using the two models, we investigate the difference and relation between the different types of cardiac alternans.

\subsection{APD alternans}

Results in Figure 2 show a steep slope of the APD restitution curve for the TP model, and a flat one for the ORd model, with maximum slopes of 1.82 and 0.35 respectively. 

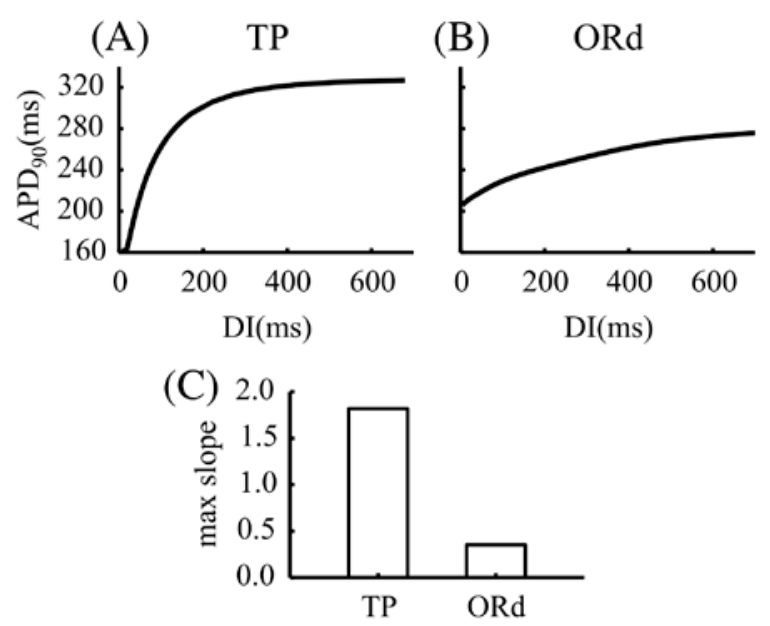

Figure 2. APD restitution curve for the TP model (A) and the ORd model (B); Maximum slopes of the APD restitution curve of the two models (C).

These results are consistent with experimental data: obvious APD alternans could occur with a steep APD restitution curve (the TP model), whereas CaT alternans could still appear when the slope of APD restitution curve is flat (the ORd model). By analyzing $\mathrm{I}_{\mathrm{Na}}$ current (not shown here) of the two models, we found that the time constant of inactivation gate of $\mathrm{I}_{\mathrm{Na}}$ in the TP model is greater than that in the ORd model. This leads to a slower recovery of $\mathrm{I}_{\mathrm{Na}}$ current in the TP model and results in a smaller next action potential. Simulation results suggest that the slope of the APD restitution curve is a crucial factor for APD alternans but has little effect on CaT alternans.

\subsection{CaT alternans}

The genesis mechanism of CaT alternans is considered to have a strong correlation with SR content. Figure 3(AB) shows the systolic intracellular $\mathrm{Ca}^{2+}$ concentration as a function of SR content of the two models. For the TP model, the maximum slope of the curve is only 1.05, resulting in no obvious CaT alternans. For the ORd model, CaT alternans did not start before the SR content reached $3.3 \mathrm{mM}$. With the accumulation of $\mathrm{Ca}^{2+}$ in $\mathrm{SR}$ content, the maximum slope of the CaT-SR curve suddenly increased to 10.04 and $\mathrm{CaT}$ oscillations appeared at the same time.

Results demonstrate the importance of the instability of intracellular $\mathrm{Ca}^{2+}$ in generating $\mathrm{CaT}$ alternans. After SR content reached a threshold, there will be a stiffer relationship between $\mathrm{CaT}$ and SR content. In this circumstance, a small variation of SR content results in a large difference in $\mathrm{CaT}$ amplitude. If the myocytes are paced at fast rates, the balance between SR release and

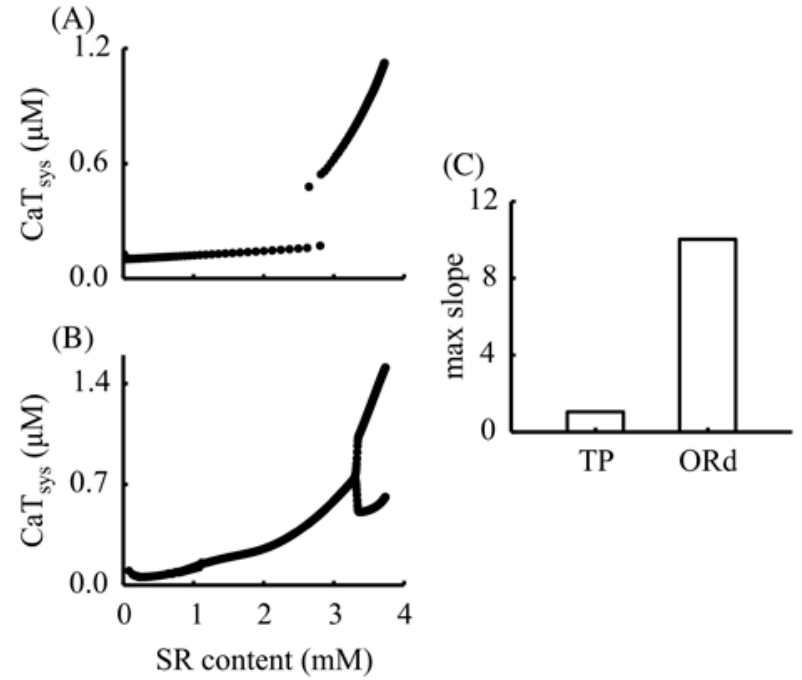

Figure 3. CaT-SR content curve for the TP model (A) and the ORd model (B); Maximum slopes of the CaT-SR content curve of the two models (C).

uptake can be easily disturbed, eventually giving rise to CaT alternans.

\subsection{Role of ICaL current}

In order to understand the functional role of $\mathrm{I}_{\mathrm{CaL}}$ on genesis of cardiac alternans, $\mathrm{I}_{\mathrm{CaL}}$ clamp protocols described in section 2 were implemented in the ORd model. The model was simulated for $5 \mathrm{~s}$ using different stimuli until it reached a stable state showing either alternans (Figure 4(A, B) or non-alternans (Figure 4(C, $D)$ ), and then $\mathrm{I}_{\mathrm{CaL}}$ current was clamped using previously recorded current traces (Figure 4(A, C) with I I CaL_alter and Figure 4(B, D) with I CaL_normal . Since all the cells were clamped with identical $\mathrm{I}_{\mathrm{CaL}}$ currents from beat-to-beat, there was no $\mathrm{I}_{\mathrm{CaL}}$ difference after $\mathrm{t}=5 \mathrm{~s}$. Results showed that in the case where $\mathrm{CaT}$ alternans was observed, clamping the model with $\mathrm{I}_{\mathrm{CaL} \_a l t e r}$ still produced alternans (Figure 4A), suggesting that $\mathrm{I}_{\mathrm{CaL}}$ alternans is not required for producing $\mathrm{CaT}$ alternans. However, $\mathrm{I}_{\mathrm{CaL} \_ \text {normal }}$ current clamp made CaT alternans disappear quickly (Figure 4B), indicating that the amplitude of $\mathrm{I}_{\mathrm{CaL}}$ is critical to $\mathrm{CaT}$ alternans. Results shown in Figure 4 C-D further demonstrated this observation. When the non-alter system was clamped with $\mathrm{I}_{\mathrm{CaL} \_a l t e r}$, it started $\mathrm{CaT}$ oscillations immediately (Figure 4C), whereas no changes were observed in the ICaL_normal clamp simulation (Figure 4D). As explained in section 3.3, since the SR content of nonalter system had reached the threshold, a large $\mathrm{I}_{\mathrm{CaL}}$ current could easily disturb the balance of the intracellular $\mathrm{Ca}^{2+}$ cycling process. Therefore, CaT alternans can be sustained relying on intrinsic properties without alternations of $\mathrm{I}_{\mathrm{CaL}}$ current. 

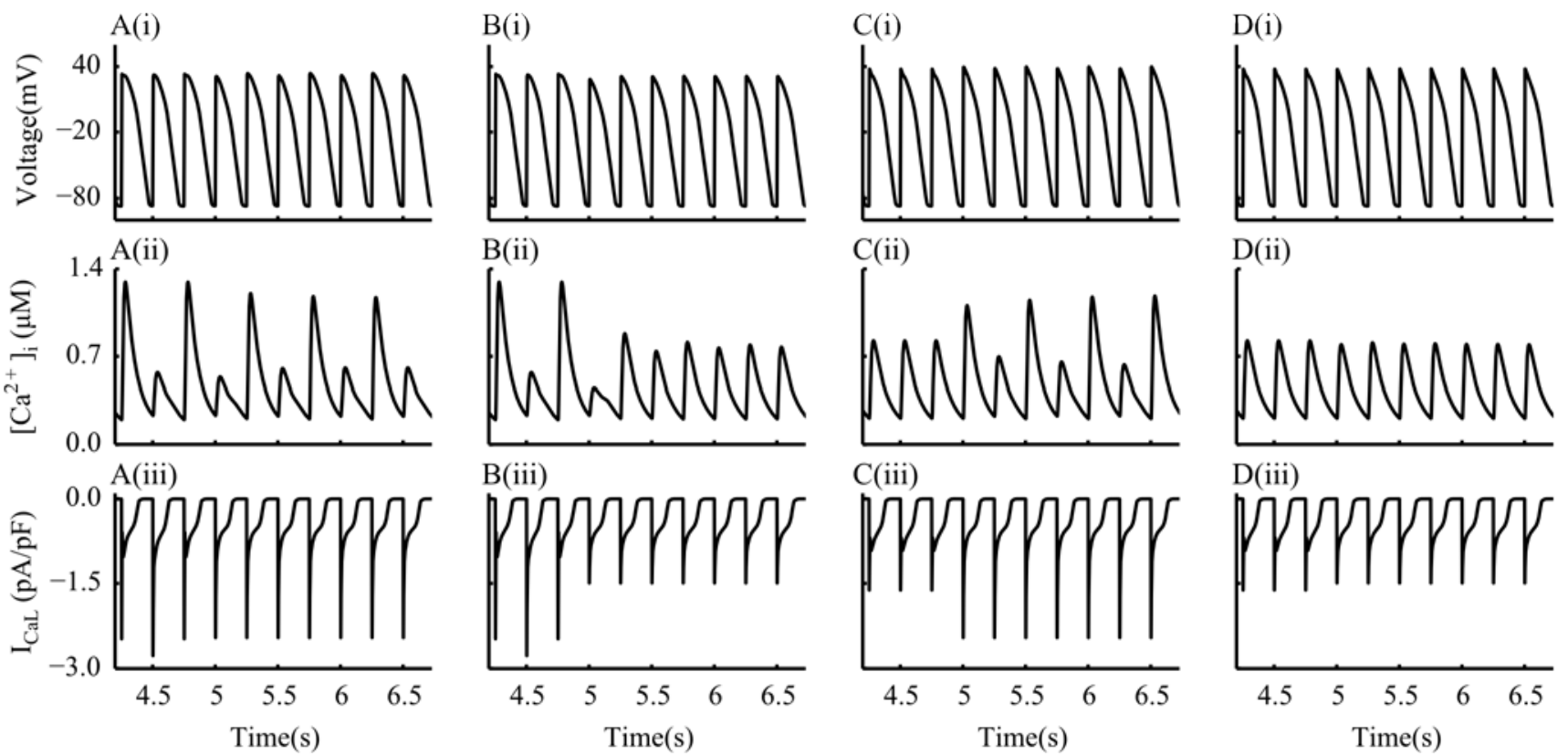

B(ii)

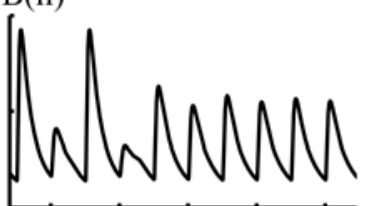

C(ii)

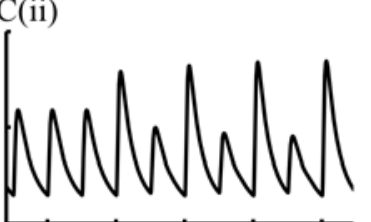

B(iii)
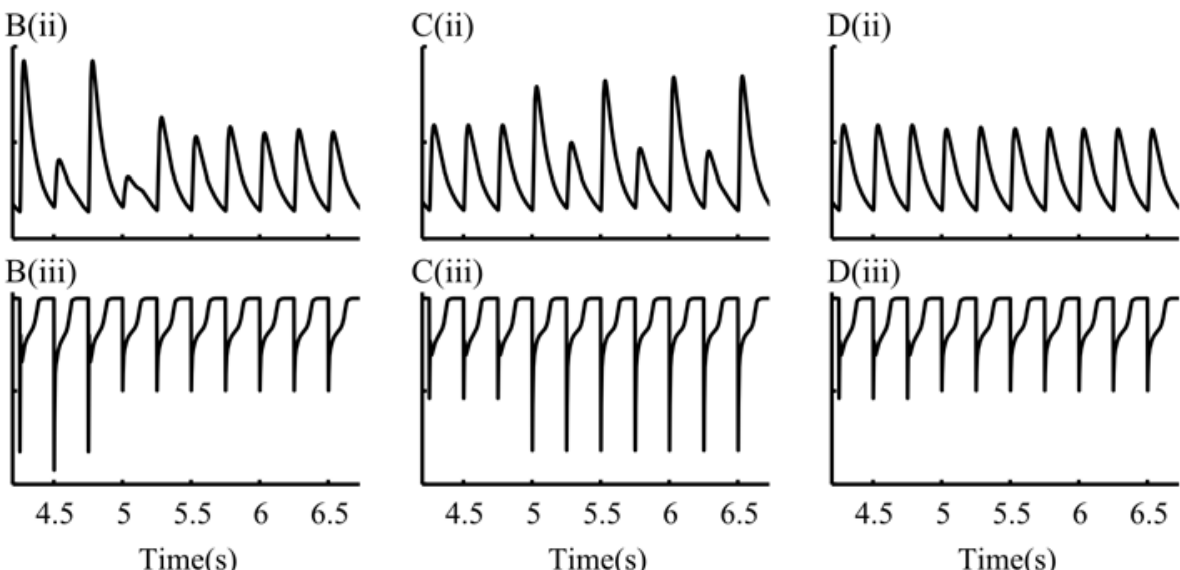

Figure 4. AP (A(i)-D(i)), CaT (A(ii)-D(ii)) and $\mathrm{I}_{\mathrm{CaL}}(\mathrm{A}(\mathrm{iii})-\mathrm{D}(\mathrm{iii}))$ time traces for $\mathrm{I}_{\mathrm{CaL}}$ clamp simulation results: (A(i-iii)) alter system clamped by $\mathrm{I}_{\mathrm{CaL} \_a l t e r}$; (B(i-iii)) alter system clamped by $\mathrm{I}_{\mathrm{CaL} \_n o r m a l}$; (C(i-iii)) non-alter system alternans clamped by ICaL_alter; (D(i-iii)) non-alter system clamped by ICaL_normal.

\section{Discussion and conclusion}

In this study, we used two human ventricle models to investigate the two types of cardiac alternans. Simulation results showed that these two models could generate cardiac alternans with completely different characteristics, which enabled us to dissect APD alternans from CaT alternans and investigate them seprately.

For APD alternans, the refractory excitability of the myocyte plays an important role. This is related to the steepness of the APD restitution curve and the recovery ability of $\mathrm{I}_{\mathrm{Na}}$ current, which determines the peak value and APD of the next AP. Results show only APD alternans cannot sustain without triggering $\mathrm{CaT}$ alternans. For $\mathrm{CaT}$ alternans, intracellular $\mathrm{Ca}^{2+}$ handling dynamics is more crucial. Simulations indicate $\mathrm{CaT}$ alternans occurs only when the SR content reaches a threshold and it can last depending on its own properties without significant APD alternans. Furthermore, I $\mathrm{I}_{\mathrm{CaL}}$ clamp simulations imply that it is the magnitude of $\mathrm{I}_{\mathrm{CaL}}$ current not the alternans of it that give rise to $\mathrm{CaT}$ alternans.

In conclusion, our results suggest that both APD and CaT alternans have their own primary mechanisms, and sustained CaT alternans is not necessarily related to APD alternans. These findings are consistent with previous experimental studies, providing a theoretical explanation for experimental phenomena, and thus helping elucidate the mechanism of cardiac alternans.

\section{Acknowledgements}

This work was supported by President's Doctoral Scholar Awards of the University of Manchester.

\section{References}

[1] Nolasco JB, Dahlen RW. A graphic method for the study of alternation in cardiac action potentials. J Appl Physiol 1968;25:191-6.

[2] Goldhaber JI, Xie L-H, Duong T, Motter C, Khuu K, Weiss JN. Action potential duration restitution and alternans in rabbit ventricular myocytes: the key role of intracellular calcium cycling. Circ Res 2005;96:459-66.

[3] H. Saitoh, J. C. Bailey, and B. Surawicz, "Alternans of action potential duration after abrupt shortening of cycle length: differences between dog Purkinje and ventricular muscle fibers," Circ. Res., vol. 62, no. 5, pp. 1027-1040, May 1988.

[4] K. H. W. J. ten Tusscher. Alternans and spiral breakup in a human ventricular tissue model. AJP Heart Circ. Physiol., vol. 291, no. 3, pp. H1088-H1100, May 2006.

[5] I. Adeniran, A. E. Harchi, J. C. Hancox, and H. Zhang. Proarrhythmia in KCNJ2-linked short QT syndrome: insights from modelling. Cardiovasc. Res., vol. 94, no. 1, pp. 66-76, Apr. 2012.

[6] O’Hara T, Virág L, Varró A, Rudy Y. Simulation of the Undiseased Human Cardiac Ventricular Action Potential: Model Formulation and Experimental Validation. PLoS Comput Biol 2011;7:e1002061.

[7] Díaz ME, O’Neill SC, Eisner DA. Sarcoplasmic Reticulum Calcium Content Fluctuation Is the Key to Cardiac Alternans. Circ Res 2004;94:650-6. 